\title{
Acknowledgement to Reviewers of Agronomy in 2017
}

\author{
Agronomy Editorial Office \\ MDPI AG, St. Alban-Anlage 66, 4052 Basel, Switzerland
}

Published: 22 January 2018

Peer review is an essential part in the publication process, ensuring that Agronomy maintains high quality standards for its published papers. In 2017, a total of 88 papers were published in the journal. Thanks to the cooperation of our reviewers, the median time to first decision was 23.5 days and the median time to publication was 53 days. The editors would like to express their sincere gratitude to the following reviewers for their time and dedication in 2017:

Abraham, E. M.
Accinelli, Cesare
Afshar, Reza Keshavarz
Alagarswamy, G.
Alakukku, Laura
Amirkhani, Masoume
Andreasson, Erik
Armstead, Ian
Ballester, Carlos
Ballesteros, Rocio
Ballizany, Wouter
Barbera, Antonio Carlo
Barrow, C. J.
Bažok, Renata
Beaver, James S.
Beckles, Diane
Belesky, David
Bellaloui, Nacer
Below, Frederick
Bertea, Cinzia
Bertoft, Eric
Bita, Craita E
Bogdan, Ileana
Borisjuk, Nikolai
Bowerman, Andrew
Boye, Kristin
Bronson, Kevin F.
Cammarata, Kirk
Cardoso, Susana
Carlsson, Anders S.
Caruso, Gianluca
Cesco, Stefano
Cheng, Xiaoyan
Cherney, Jerry H.
Cihacek, Larry
Cirilli, Marco

\author{
Magarey, Roger \\ Mahmood, Khalid \\ Marchetti, Marta \\ Marla, Sandeep \\ Marley, Christina \\ Marocco, Adriano \\ Martínez, Luis Carlos \\ Martinez, Manuel \\ Maserti, Bianca Elena \\ Masuda, Shinji \\ Mathews, Shiny \\ Matiu, Michael \\ Matos, Ana Rita \\ McCollum, Daniel W. \\ Medina Piles, Vicente \\ Meijer, H.J.G. \\ Mennan, Hüsrev \\ Merah, Othmane \\ Migliaccio, Kati W. \\ Moar, William J. \\ Monteiro, Filipa \\ Montgomery, James A. \\ Moreno, Miguel \\ Mori, Kazuhiro \\ Moriguchi, Takaya \\ Moriondo, M. \\ Myers, Alan \\ Nakashima, Kazuo \\ Nam, Kyoung Hee \\ Nandwani, Dilip \\ Nardi, Serenella \\ Nicoletto, Carlo \\ Norton, Gareth J. \\ Nurse, Rob \\ Ober, Eric \\ Oenema, Oene
}


Conner, Robert

Copeland, Les

Crews, Timothy E.

Dardick, Christopher D.

Daugrois, Jean-Heinrich

De La Varga, Herminia

Délano-Frier, John Paul

Delgado, Antonia M. Rojano

Deligios, Paola A.

D'hulst, Christophe

Dodd, Ian C.

Döring, Thomas

Duiker, Sjoerd

Ebdon, Scott

Edae, Erena A.

Egan, Martin

Egerton-Warburton, Louise

El-Seedi, Hesham R.

Emes, Michael

Engelsdorf, Timo

Ergon, Åshild

Evangelisti, Edouard

Fagnano, Massimo

Fares, Clara

Fasoula, Vasilia

Fernandez-Delgado Juarez, Marina

Ferrante, Antonio

Fischer, Klara

Fotopoulos, Vasileios

Fox, William E.

Fragkostefanakis, Sotirios

Franco, Albina Cristina Ribeiro

Fujita, Masayuki

Galek, Renata

Gao, San-Ji

Gerhards, Roland

Ghamkhar, Kioumars

Ghasemi Pirbalouti, Abdollah

Giroux, Mike

Gömöryová, Erika

Gong, Xue

Greer, Mitchell

Gutiérrez, Edgar

Haghverdi, Amir

Hasegawa, Paul Michael

Hasegawa, Toshihiro

Hatfield, Jerry

Hazak, Ora

$\mathrm{He}$, Jie

Hein, Gary

Herny, Robert

Herrero, Baudilio
Olfs, Hans-Werner

Olin, Stefan

Orzolek, Michael D.

Osroosh, Yasin

Pandey, Avinash Chandra

Pandolfini, Tiziana

Payn, Tim

Penn, Chad

Perez De La Vega, Marcelino

Persson, Tomas

Pierik, Ronald

Pinto, Victor

Pitt, John

Pizzeghello, Diego

Portugal, João

Price, Adam

Prochaska, Charikleia

Przybysz, Arkadiusz

Puppala, Naveen

Puri, Akshit

Rallo, Giovanni

Rapacz, Marcin

Rehrig, Erin M.

Revilla, Pedro

Riday, Heathcliffe

Rocateli, Alexandre

Rodríguez-Seijo, Andres

Rohila, Jai.

Roldán-Cañas, José

Ruiz-Altisent, Margarita

Ruiz-Sánchez, Ma Carmen

Sacchi, Gian Attilio

Sagi, Moshe

Saleem, Muhammad

Samac, Deborah A.

Sangireddy, Sasikiran Reddy

Sappington, Tom

Satbhai, Santosh

Schepers, James S.

Schipper, L.A.

Schmidt, Hans-Peter

Schmittgen, Simone

Scholz, Miklas

Schultz, Richard C.

Shahba, Mohamed A.

Shavrukov, Yuri

Shen, Qirong

Sheng, Wenyi

Shockey, Bill

Sieling, Klaus

Sims, Brian

Singh, Bal Ram 
Hiltbrunner, Andreas

Hoagland, Lori A.

Hsiao, Theodore C.

Huang, Lei

Idrissi, Omar

Impullitti, Ann

Isakeit, Thomas

Iseki, Kohtaro

Ishii, Yasuyuki

Jahanzad, E.

Jeong, Changyoon

Jessup, Russell W.

Jian, Fuji

Jidda, M. Bukar

Jin, Zhao

Jones, Chris $S$.

Jung, Chol-Hee

Kalaji, Hazem Mohamed

Kaleita, Amy

Kamiya, Takehiro

Katsantonis, Dimitrios

Kaur, Gurpreet

Kavvadias, Victor

Keech, Olivier

Keene, Clair

Khan, Habib

Kirkham, M. B.

Klasson, Thomas

Klimeski, Aleksandar

Kluepfel, D. A.

Knapp, Corrine

Knaus, Ulrich

Knicky, Martin

Kosova, Klara

Kotcon, James B.

Kozaki, Akiko

Krishnan, Sanalkumar

Larson, Steven R.

Liang, Xi

Ligterink, Wilco

Lima, Giuseppina Pace P.

Lin, Amy Hui-Mei

Liu, Haibo

Liu, Shuyu

Lobry De Bruyn, Lisa

Longo, Laura

Longstaffe, James G.

Lupin, Antonio

Lyons, Eric M.

Ma, Lena

Macnack, Natasha
Singh, Jugpreet

Sirsat, Sujata

Skeffington, Richard

Soanes, Darren

Song, Guo-qing

Soots, Kaarel

Sorrenti, Giovambattista

Speratti, Alicia B.

Spokas, Kurt

Steele, Katherine A.

Stelinski, Lucasz

Stoddard, Fred

Storer, Nicholas P.

Tacke, Rebecca

Tecco, Nadia

Teodor, Rusu

Thammina, Chandra

Theurl, Michaela

Pórarinsdóttir, Ragnheiður

Tian, Bin

Toselli, Moreno

Trenchard, Liz

Trenholm, Laurie E.

Trögl, Josef

Trout, Thomas

Trueman, Stephen

Tsagarakis, Konstantinos

Tuinstra, Mitch

Turner, Neil

Tyson, Julian

Uphoff, Norman

Valenzuela, Hector

Van Meerbeek, Koenraad

Vanderborght, Jan

Vaz Moreira, Ivone

Vleugels, Tim

Volaire, Florence

Wallach, Daniel

Waller, Uwe

Ward, James

Wilson, Houston

Wu, Jianshuang

$\mathrm{Wu}$, Jixiang

Xuan, Tran Dang

Yang, Fei

Yavitt, Joseph

Yu, Kang

Zanin, Laura

Žiarovská, Jana

Ziska, Lewis $\mathrm{H}$. 
(C) 2018 by the author. Licensee MDPI, Basel, Switzerland. This article is an open access article distributed under the terms and conditions of the Creative Commons Attribution (CC BY) license (http://creativecommons.org/licenses/by/4.0/). 